This is a self-archived version of an original article. This version may differ from the original in pagination and typographic details.

Author(s): Ala-Kitula, Anniina; Peltonen, Jussi; Finni Juutinen, Taija; Linnamo, Vesa

Title: Physical activity on days with and without soccer practice in 12-13-year-old boys

Year: 2019

Version: Accepted version (Final draft)

Copyright: (c) 2019 Taylor \& Francis

Rights: In Copyright

Rights url: http://rightsstatements.org/page//nC/1.0/?language=en

Please cite the original version:

Ala-Kitula, A., Peltonen, J., Finni Juutinen, T., \& Linnamo, V. (2019). Physical activity on days with and without soccer practice in 12-13-year-old boys. Science and Medicine in Football, 3(3), 245250. https://doi.org/10.1080/24733938.2018.1562276 


\section{Physical Activity on Days with and without Soccer Practice in}

\section{2-13-year-old Boys}

Anniina Ala-Kitula ${ }^{1}$, Jussi Peltonen ${ }^{2}$, Taija Finni ${ }^{1}$ and Vesa Linnamo ${ }^{1}$

1 Neuromusuclar Research Center, Faculty of Sport and Health Sciences,

University of Jyväskylä, Jyväskylä, Finland

2 Polar Electro Oy, Jyväskylä, Finland

Anniina Ala-Kitula, M.Sc., Vuokkokatu 7, 37120 Nokia, +358 50348 1049, anniina.alakitula@gmail.com.

Jussi Peltonen, Ph.D., Senior Researcher, Polar Electro Oy, Laukaantie 4, FI-40320 Jyväskylä, Finland, jussi.peltonen@polar.com, +358 407265247

Taija Finni, Ph.D., Professor, Biology of Physical Activity, University of Jyväskylä, P.O.Box 35, FI-40014 University of Jyväskylä, Finland, +358 40 5566582, taija.finni@jyu.fi

Vesa Linnamo, Ph.D., Professor, Biology of Physical Activity, University of Jyväskylä, P.O.Box 35, FI-40014 University of Jyväskylä, Finland, +358 40 5044800, vesa.linnamo@jyu.fi 


\section{Abstract}

Majority of children are unable to meet the recommended 60 minutes of daily moderate to vigorous physical activity (MVPA). Even participation into organized sport may not guarantee that recommended amount of activity is reached. Purpose of this study was to examine the amount and intensity of physical activity (PA) on days with and without practices and to find out whether daytime PA preceding soccer practice is associated with certain practice-time PA intensity level.

Daily light, moderate and vigorous PA of 18 boys between 12 to 13 years of age engaged in soccer was measured for 9 days with and without soccer practices with wrist-worn Polar A300 activity meter. In addition, PA during practices was extracted and compared to PA preceding the practice session.

The daily MVPA was twice as high on days with training as compared to days without training both on weekdays (mean difference $\pm 95 \%$ confidence interval $=67 \mathrm{~min} \pm 14 \mathrm{~min})$ and on weekend $(63 \mathrm{~min} \pm 36$ $\mathrm{min})$. PA preceding soccer practice had a positive correlation with all PA intensity levels during practice $(r=0.34-0.84, \mathrm{p}<0.05-0.01)$.

Physical activity should be promoted especially on days without organized practices and children should be encouraged to be physically active throughout the day as this was not found to compromise their activity during training.

\section{Introduction}

It is widely known that children's physical activity (PA) level varies during the day (e.g. De Baere, Lefere, De Martelaer, Philippaerts, \& Seghers 2015; Cox, Schofield, Greasley, \& Kolt 2006; van Stralen et al. 2014). In general children are more physically active outside school days and most of their moderate-tovigorous physical activity (MVPA) is accumulated during leisure time (e.g. Beck, Chard, Hilzendegen, Hill, \& Stroebele-Benschop, 2016). There is also a considerable variation in PA between children during the school days, on hours after school as well as during the weekends (e.g. Beck et al. 2016, Fairclough et 
al. 2014 and Hardman et al. 2009).

Growing majority of children around the world is not able to meet the recommended amounts of children's daily physical activity (e.g. Kokko \& Mehtälä, 2016; Kokko \& Hämylä, 2015; Katzmarzyk et al., 2014). In Finland, the recommended amount of PA for children between 7 and 12 years of age is from 90 to 120 minutes and for children between 13 and 18 years of age the recommendation is from 60 to 90 minutes (Ministry of Education and Culture, 2016). From these times, at least half should be accumulated from moderate intensity level. According to Kokko \& Mehtälä (2016) only 20\% of Finnish children are meeting the recommendations. In USA and Canada, the recommendations for physical activity state that children between ages 6 and 17 should have at least 60 minutes of MVPA daily (The Canadian Society for Exercise Physiology, 2016; The U.S. Department of Health and Human Services, 2008). According to Katzmarzyk et al. (2014) only $22 \%$ of children in the U.S. are meeting the recommendations.

Participation in organized sport has been offered as one possible solution by American Academy of Pediatrics to increase children's daily physical activity (Committee on Sports Medicine and Fitness and Committee on School Health, 2001). However, it is not guaranteed that participation into organized sport alone provides enough PA. For example, study by Leek et al. (2011) indicated that no more than one third of youth athletes reached the recommended 60 minutes of MVPA during soccer practices.

This study measured the intensity and amount of PA during 9 consecutive days from 12-13-year-old boys who participated in soccer practise three times/ 
week. The primary purpose was to quantify the difference in PA between the days with and without practices. As it's possible, but to our knowledge not reported elsewhere, that PA during organized sport is influenced by some level of PA (i.e. sedentary, low, moderate or vigorous) during the day before the practice session, the secondary purpose was to investigate whether individual's preceding PA on the practice day is associated with PA during soccer practice. 


\section{Methods}

\section{Participants}

Children were recruited from a boys' junior soccer team in central Finland.

Twenty-one children volunteered to participate, and they were able to quit at any point at will without any consequences. Parents signed the informed consent and the study followed the principles of the Declaration of Helsinki. Total of 18 children returned their diary and had sufficient amount of PA data and their data were included in the analysis. The mean age at the beginning of the measurement was $12.6 \pm 0.3$ years. Mean height was $156.5 \pm 7.5 \mathrm{~cm}$ and body mass $47.3 \pm 8$ $\mathrm{kg}$.

\section{Study procedures}

Subjects were instructed to wear Polar A300 -activity monitors (Polar Electro Oy, Finland) in the wrist of their non-dominant hand during day and night for 9 consecutive days in February 2017 starting on Monday. During soccer practices subjects wore also H7 heart rate monitors (Polar Electro Oy, Kempele, Finland). Soccer practices took place on Monday, Wednesday, Saturday and again Monday totalling to three training sessions on weekdays and one on weekend. Each of the 18 subjects was present in at least one of the training sessions. Attendance rates of the soccer practices were $83 \%, 78 \%, 78 \%$ and $83 \%$.

The practice sessions were not controlled by the researchers, and the coaches followed their pre-planned practice programmes. Even though the coaches were aware of the study, it did not influence their planning. Structure of 
the training sessions was following: The first third consisted of small-sided soccer games (individual playing area $35-55 \mathrm{~m}^{2}$ ), with reduced number of players (4 vs. 4) and without goals or goalkeepers. The second third consisted of game episodes with close to actual game dimensions, goals and goalkeepers (individual playing area $140-200 \mathrm{~m}^{2}$ ). The last third consisted of small-sided soccer games with goalkeepers and close to real-game dimensions (individual playing area 60-120 $\mathrm{m}^{2}$ ). One practice session lasted on average 90 minutes.

\section{Assessments and data reduction}

Physical activity intensity was obtained automatically by A300 and recorded in metabolic equivalents (MET's). PA intensity was derived from accelerometer signal using Polar's proprietary algorithm, which has been previously validated against indirect calorimetry (Kinnunen et al. 2012). In soccer practices, also H7 heart rate monitor was worn. When heart rate monitor is on, A300 checks heart rate and acceleration based PA intensity every $30 \mathrm{~s}$. If PA intensity is medium or high, recorded PA intensity is taken from heart rate data (Hiilloskorpi et al. 1999) and if PA intensity is low or subject is at rest or sitting, PA intensity is taken from accelerometer data. One PA intensity sample was recorded every $30 \mathrm{~s}$. The days and times of soccer practice were marked down so that practice intervals could be distinguished afterwards from the PA data. After the 9-day period the PA data were collected for further analysis.

Recorded MET values were allocated to three categories according to World Health Organisation (2015), light (LPA), moderate (MPA) and vigorous (VPA) physical activity, and with following limits: 1.8 MET $<$ LPA $<=3.5$ MET; 
3.5 $\mathrm{MET}<\mathrm{MPA}<=6.0 \mathrm{MET}$; and 6.0 MET < VPA. The results were expressed as cumulative minutes in LPA, MPA, VPA levels for various time periods that are explained below.

Physical activity was analysed from 7 am to 9 pm according to Trost et al. (2000). Difference between training vs. non-training day was calculated for two consecutive days. As training day always preceded non-training day, daily window was further divided to first (from 7 am to $2 \mathrm{pm}$ ) and second (from $2 \mathrm{pm}$ to $9 \mathrm{pm}$ ) halves to check that first half, which was free of organised sport, did not demonstrate any systematic difference between the days. Eight days out of nine were included in the analysis and one day was excluded as it followed another non-training day. Subjects that were absent from evening's training sessions were excluded from that and the following days analysis but were included again when they were present in practices. Thus, each subject may appear in weekdays analysis 1,2 or 3 times, depending on their presence in trainings.

To analyse individually whether PA preceding practice may influence PA during practice, we collected each subject's PA data separately based on timestamp when the practice was started and not on fixed timeframe. As practices started either at 4:15 pm or 7:15 pm, PA data window size before the practice varied between days.

\section{Statistical analysis}

Statistical calculations were made with Apple iWork Numbers version 4.1 and with IBM SPSS Statistics version 24. The data were normally distributed according to Shapiro-Wilk test and parametric tests were chosen. The differences 
in PA at a given intensity level between days with and without practices were analysed with paired t-tests. To test whether PA preceding practice was associated with PA during the practice Pearson's correlation coefficients were calculated for each PA intensity level separately for weekday and weekend day practice sessions. The level of significance was set to 0.05 .

\section{Results}

Training vs. non-training day comparison was based on practice attendance and consisted of $43(14+14+15)$ samples from weekdays, including all 18 subjects at least once, and 14 samples from weekend.

During weekdays, PA was higher during training day than non-training day at MPA (mean difference $\pm 95 \%$ confidence interval $=27 \pm 10 \mathrm{~min})$ and VPA $(40$ $\pm 7 \mathrm{~min}$ ) levels but lower at LPA (-28 $\pm 19 \mathrm{~min})$ level (Table 1). There wasn't significant difference at any PA level during the first half of the day indicating lack of bias between training and non-training days. In contrast, increased PA was observed during the second half of the day at MPA (27 $\pm 7 \mathrm{~min})$ and VPA ( $37 \pm 7$ min) levels and decreased activity at LPA (-18 $\pm 12 \mathrm{~min})$ level.

During weekend, PA was higher during training day at MPA (20 $\pm 19 \mathrm{~min})$ and VPA (43 \pm 26 min) levels but no significant difference was observed at LPA level. Weekend PA was not divided to two halves as the divided PA data were not normally distributed. 
[Table 1 near here]

Table 1. Average physical activity time at different intensity levels (LPA = light, $\mathrm{MPA}=$ moderate, $\mathrm{VPA}=$ vigorous, $\mathrm{MVPA}=$ moderate to vigorous) on weekdays with and without practices. $\mathrm{SD}=$ standard deviation; $\mathrm{CI}=$ confidence interval. $\mathrm{N}$ $=43$.

[Table 2 near here]

Table 2. Average physical activity time at different intensity levels (LPA $=$ light, $\mathrm{MPA}=$ moderate, $\mathrm{VPA}=$ vigorous, $\mathrm{MVPA}=$ moderate to vigorous) on weekend days with and without practices in minutes. $\mathrm{SD}=$ standard deviation; $\mathrm{CI}=$ confidence interval. $\mathrm{N}=14$.

Combined amount of MVPA on weekdays was $143 \pm 32($ mean \pm SD) minutes with practices and $76 \pm 38$ minutes without practices. On weekend, the corresponding numbers were $118 \pm 36$ and $56 \pm 49$ minutes. The lowest recommended amount of daily MVPA (> $60 \mathrm{~min}$ ) was reached by $100 \%$ vs. $67 \%$ of the participant (training vs. non-training days) during weekdays and $100 \%$ vs. $36 \%$ during weekends.

Illustration of LPA, MPA and VPA by every hour from 7 am to $9 \mathrm{pm}$ during training and non-training days is displayed in Figure 1.

[Figure 1 near here]

Figure 1. Illustration of daily physical activity from 7 am to $9 \mathrm{pm}$. First column represents training days $(\mathrm{a}, \mathrm{c}, \mathrm{e}, \mathrm{g})$ and second column non-training days $(\mathrm{b}, \mathrm{d}, \mathrm{f}$, h). Data in first three rows (from a to $\mathrm{f}$ ) are from weekdays and in bottom row ( $\mathrm{g}$ and $\mathrm{h}$ ) from weekend. Colours are: blue $=$ LPA, cyan $=$ MPA, yellow $=$ VPA.

Physical activity preceding the practices had significant positive correlations with different activity levels during practices on both on weekdays 
and weekend days. For weekday correlation three weekdays were combined (Table 3) and for weekend day correlation only one day was used (Table 4). The strongest correlation between preceding physical activity on the same day and practice time PA was found between preceding LPA on the same day and MPA during practices both on weekdays $(r=0.745, \mathrm{p}=0.000)$ and on a weekend day $(\mathrm{r}$ $=0.837, \mathrm{p}=0.000)$.

[Table 3 near here]

Table 3. Correlation coefficients (r) between weekday physical activity levels during practices and total amount of physical activity before practice on the same day. $*=\mathrm{p}<0.05 ; * *=\mathrm{p}<0.010 ; * * *=\mathrm{p}<0.001 .95 \%$ confidence interval upper and lower limits is expressed in brackets. $\mathrm{N}=43$.

[Table 4 near here]

Table 4. Correlation coefficients (r) between weekend physical activity levels during practices and total amount of physical activity before practice on the same day. $*=\mathrm{p}<0.05 ; * *=\mathrm{p}<0.010:^{* * *}=\mathrm{p}<0.001 .95 \%$ confidence interval upper and lower is expressed in brackets. $\mathrm{N}=14$.

\section{Discussion}

Purpose of this research was to examine what is the amount of PA on days without practice compared to days with practice. Purpose was also to study the relationship of preceding physical activity with the activity during soccer practice of the same day with children. The main findings of the present study were that in a sample of boys participating to soccer 1) the amount of MVPA attained on practice days is not achieved on days without practice, 2) the MVPA recommendations were not met on weekend days without practice and 3) previous 
PA of the same day before soccer practice has positive correlation with PA during soccer practice at several different activity levels.

\section{Physical activity on days with and without practices}

The total amount of physical activity was lower on days without practice both on weekdays and weekend days, which was expected based on previous studies ( Hebert, Møller, Andersen, \& Wedderkopp, 2015; O’Neill, Pate, \& Hooker, 2011; Wickel \& Eisenmann, 2007). In the light of recommendations for physical activity, in which the lower limit for daily MVPA is 60 minutes, the amount of MVPA on weekend days without practice was below the recommendation in $67 \%$ of the observations. Previous studies among children by Wickel and Eisenmann (2007) and O'Neill et al. (2011) have indicated that the increased amount of MVPA on days with organized sport is not maintained on days without organized sport. They have suggested that the MVPA on practice days is replaced by LPA on days without practice. In the current study, increase in MVPA due to practice during weekdays $(67 \pm 14 \mathrm{~min})$ was much higher than decrease in LPA $(-28 \pm 19$ min) (Table 1). Therefore, it seems that the increased MVPA time on practice days is shifted to sedentary time on days without practice.

\section{Acute preceding activity and general activity level}

In the current study, there were significant correlations on different activity levels between acute preceding activity on the same day and activity during practices. The strongest associations were between preceding light physical activity and moderate physical activity during practice. Craft et al. (2012) have found evidence 
with adults, that performing sustained physical activity on moderate to vigorous activity level does not reduce the time spent sedentary. Children with high PA level are known to be active throughout the day compared to less active children (Fairclough et al. 2012), but previous studies have not indicated whether there would be any correlation between physical activity at given activity level before practice and physical activity during the practice. Results from this study suggest that light physical activity before practice may lead to increased physical activity on the moderate activity level during practices. So being physically active at light activity level before the practice on the same day may promote more moderate physical activity in practices. One way to interpret the correlation coefficients is also to look them in the perspective of effectiveness. As LPA before practice seems to increase MPA during the practice, could the information on subject's PA before practice be used by coach to evaluate what kind of practice would be effective and meaningful on that day?

\section{Methodological considerations}

Assessment of daily PA intensity may be compromised if disciplines vary considerably from day to day. This is because algorithm in the activity monitor does not know what discipline is undertaken. Thus running, cycling, swimming and muscle strength training may all produce very different estimates of PA intensity. However, this probably did not interfere with the conclusion of the current study, that children were less active during days without practice, because it is unlikely that children have spent large fraction of their daily activities in swimming and cycling in Finland mid-February, or in strength training exercises 
due to their young age. In soccer, heart rate monitoring was the preferred method to assess PA intensity due to linear relationship between heart rate and energy expenditure, especially on high intensities. Expanding heart rate monitoring to activities outside soccer practices was also considered. Eventually, it was deemed unpractical with the technology in use (H7 heart rate monitor) and possibly erroneous because heart rate is also affected by psychological factors such as excitement and stress. Both heart rate monitors and wrist-worn accelerometers have been previously validated as reliable methods to assess energy expenditure during physical activities (Hiilloskorpi et al. 1999; Kinnunen et al. 2012).

\section{Limitations of the study}

Generalisation of the study results is limited, since this study had only 18 participants. Also, this study did not have a control group and therefore it was not possible to compare the results to children with equal demographic values but not involved in soccer practices. However, as the current study wasn't an intervention study - participant had voluntarily decided to play soccer for years - the benefits of control group in this kind of setup are limited. For example, inclusion of control group would not solve causality question: Did participation to organised sport cause activity or did activity cause participation to organised sport?

It is also possible that participation into this research made children more or less active than they would have normally been. Previous studies (Brusseau, Kulinna, Kloeppel, \& Ferry, 2012; Goodman, Paskins, \& Mackett, 2012; Hardman, Horne, \& Rowlands, 2009) have shown that children are less active during winter time compared to other seasons, especially spring and summer. 
Therefore, it is reasonable to question whether children would have been generally more active outside practice time if the measurements were done during spring or summer time.

\section{Conclusions}

Main finding of the current study was that children engaged in soccer club accumulate twice as much moderate-to-vigorous physical activity on days with organised soccer practices than on days without practices. On weekdays without organised soccer training, average PA was above the lowest recommended limit (60 minutes of MVPA) but failed to reach the highest recommended amount (120 minutes of MVPA). On weekend, even the lowest recommended amount of daily physical activity was met only on practice days. Secondary finding of the current study was that physical activity on the same day before practice has positive relationship with physical activity on soccer practice. In conclusion, the current findings emphasize the importance of promoting physical activity especially on days without organized practices. Soccer coaches for 12 to 13 year boys, should encourage children to be physically active throughout the day as this was not found to compromise their activity during training. The results of the current study may help parents to understand the underlying reasons why their children do or do not meet the current recommendations for daily physical activity and change their behaviour accordingly.

\section{Disclosure statement}

No potential conflict of interest was reported by the authors. 


\section{References}

De Baere, S., Lefevre, J., De Martelaer, K., Philippaerts, R., \& Seghers, J. 2015. Temporal patterns of physical activity and sedentary behavior in 10-14 year-old children on weekdays. BMC Public Health, 15, 791. doi: 10.1186/ s12889-015-2093-7

Beck, J., Chard, C. A., Hilzendegen, C., Hill, J., \& Stroebele-Benschop, N. 2016. In-school versus out-of-school sedentary behavior patterns in U.S. children. BMC Obesity, 3, 34. doi: 10.1186/s40608-016-0115-3

Brusseau, T. A., Kulinna, P.H., Kloeppel, T. \& Ferry, M. 2012. Seasonal variation of American Indian children's school-day physical activity. Biomedical Human Kinetics, 4, 82 - 87. doi: 10.2478/v10101-012-0015-z

The Canadian Society for Exercise Physiology. 2016. Canadian 24-Hour Movement Guidelines for Children and Youth. Retrieved from https:// indd.adobe.com/view/b82b4a90-6e46-4b1a-b628-d53805688baf

Committee on Sports Medicine and Fitness and Committee on School Health. 2001. Organized Sports for Children and Preadolescents. Pediatrics, 107(6), 1459-1462. doi: 10.1542/peds.107.6.1459

Cox, M., Schofield, G., Greasley, N. \& Kolt, G. S. 2006. Pedometer steps in primary school-aged children: A comparison of school-based and out-ofschool activity. Journal of Science and Medicine in Sport, 9, 91-97. doi: 10.1016/j.jsams.2005.11.003

Craft, L. L., Zderic, T. W., Gapstur, S. M., Vanlterson, E. H., Thomas, D. M., Siddique, J. \& Hamilton, M.1. 2012. Evidence that women meeting physical activity guidelines do not sit less: An observational inclinometry study. International Journal of Behavioral Nutrition and Physical Activity, 9, 122. doi: 10.1186/1479-5868-9-122

Fairclough, S. J., Beighle, A., Erwin, H., \& Ridgers, N. D. 2012. School day segmented phys- ical activity patterns of high and low active children. BMC Public Health 12, 406.

Fairclough, S. J., Boddy, L. M., Mackintosh, K.A., Valencia-Peris, A. \& RamirezRico, E. 2014. Weekday and weekend sedentary time and physical activity 
in differentially ac- tive children. Journal of Science and Medicine in Sport 18 (4), 444-449.

Goodman, A., Paskins, J. \& Mackett, R. 2012. Day Length and Weather Effects on Children's Physical Activity and Participation in Play, Sports, and Active Travel. Journal of Physical Activity and Healt, h 9(8), 1105-1116. Retrieved from https:/www.ncbi.nlm.nih.gov/pmc/articles/PMC3584676/

Hardman, C. A., Horne, P. J. \& Rowlands, A. V. 2009. Children's Pedometerdetermined Physical Activity During School-time and Leisure-time. Journal of Exercise Science and Fitness, 7(2), 129-134. doi: 10.1016/ S1728-869X(09)60016-2

Hebert J. J., Møller N. C., Andersen L. B. \& Wedderkopp N. 2015. Organized Sport Participation Is Associated with Higher Levels of Overall HealthRelated Physical Activity in Children (CHAMPS Study-DK). PLoS ONE, 10(8): e0134621. doi: 10.1371/journal.pone.0134621

Hiilloskorpi, H., Fogelholm, M., Laukkanen, R., Pasanen, M., Oja, P., Mänttäri, A., \& Natri, A. 1999. Factors affecting the relation between heart rate and energy expenditure during exercise. International journal of sports medicine, 20(07), 438-443.

Katzmarzyk, P. T., Dentro, K., Beals, K., Crouter, S., Eisenmann, J. C., McKenzie, T. L., \& Spruijt, D. 2014. The 2014 united states report card on physical activity for children and youth. National Physical Activity Plan.

Kinnunen, H., Tanskanen, M., Kyröläinen, H., \& Westerterp, K. R. (2012). Wristworn accelerometers in assessment of energy expenditure during intensive training. Physiological measurement, 33(11), 1841.

Kokko, S. \& Hämylä. R (ed.). 2015. Lasten ja nuorten liikuntakäyttäytyminen Suomessa. Valtion liikuntaneuvoston julkaisuja 2015: 2. Valtion liikuntaneuvosto.

Kokko, S. \& Mehtälä, A (ed.). 2016. Lasten ja nuorten liikuntakäyttäytyminen Suomessa. Valtion liikuntaneuvoston julkaisuja 2016:4. Valtion liikuntaneuvosto. 
Leek, D., Carlson, J. A., Cain, K. L., Henrichon, S., Rosenberg, D., Patrick, K. \& Sallis, J. F. 2011. Physical activity During Youth Sports Practices. Archives of Pediatrics and Adolescent Medicine Journal, 165(4), 294-299. doi:

10.1001/archpediatrics.2010.252

Ministry of the Education and Culture, Finland. 2016. Joy, play and doing together - Recommendations for physical activity in early childhood. Publications of the Ministry on Education and Culture, Finland 2016:21. Retrieved from http://urn.fi/URN:ISBN:978-952-263-413-9

O’Neill, J. R., Pate, R. R. \& Hooker, S. p. 2011. The contribution of dance to daily physical activity among adolescent girls. International Journal of Behavioral Nutrition and Physical Activity, 8, 87. Retrieved from http:// www.ijbnpa.org/content/8/1/87

Trost, S. G., R. R. Pate, P. S. Freedson, J. F. Sallis, and W. C. Taylor. 2000. "Using Objective Physical Activity Measures with Youth: How Many Days of Monitoring Are Needed?" Medicine and Science in Sports and Exercise 32 (2): 426-31.

The U.S. Department of Health and Human Services. 2008. 2008 Physical Activity Guidelines for Americans. Retrieved from https://health.gov/ paguidelines/pdf/paguide.pdf

van Stralen, M. M., Yıldırım, M., Wulp. A., te Velde, S. J., Verloigne, M., Doessegger, A., Androutsos, O., Kovács, É, Brug, J. \& Chinapaw, M. J. M. 2014. Measured sedentary time and physical activity during the school day of European 10- to 12-year-old children: The ENERGY project. Journal of Science and Medicine in Sport, 17(2), 201-206. doi: http://dx.doi.org/ 10.1016/j.jsams.2013.04.019

Wickel, C. E. \& Eisenmann, J. C. 2007. Contribution of Youth Sport to Total Daily Physical Activity among 6- to 12-yr-old Boys. Medicine \& Science in Sports \& Exercise, 39(9), 1493-1500. doi: 10.1249/mss. 0b013e318093f56a 
World Health Organization. 2010. Global recommendations on physical activity for health. Retrieved from http://apps.who.int/iris/bitstream/ 10665/44399/1/9789241599979_eng.pdf. 
a)

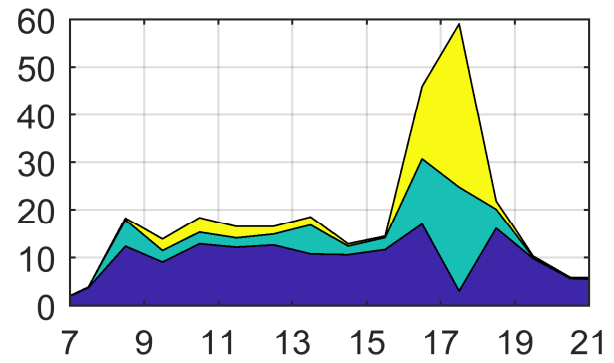

c)

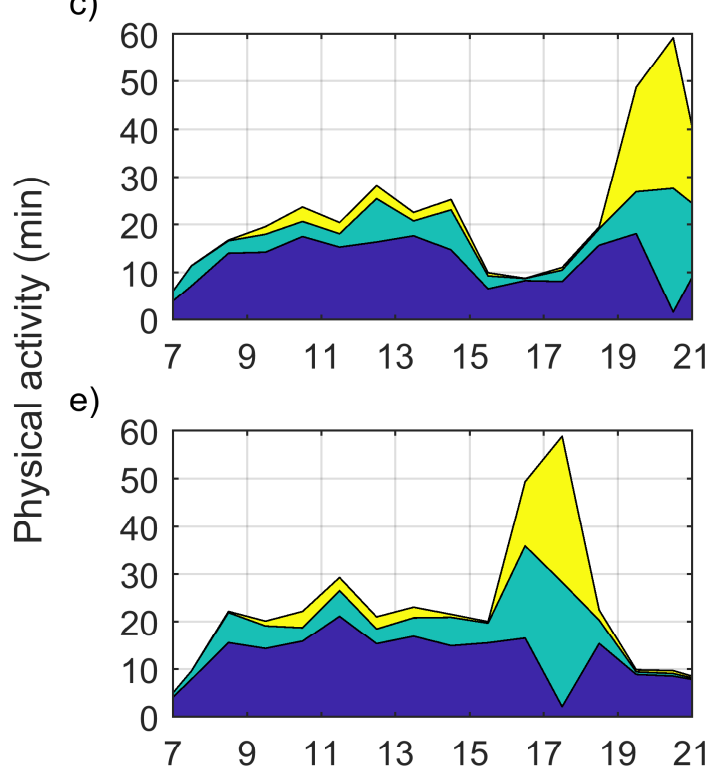

g)

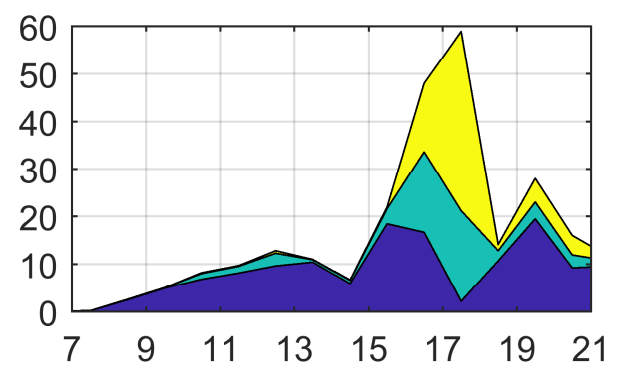

b)

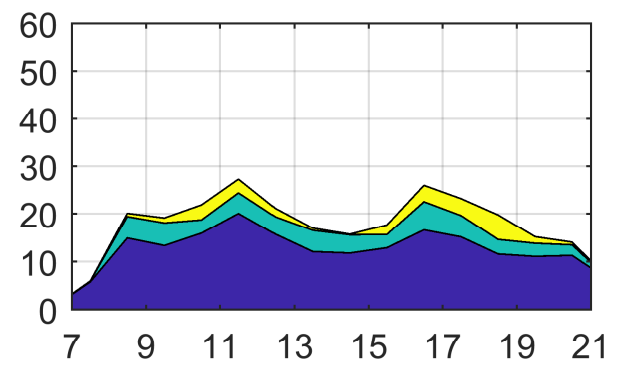

d)

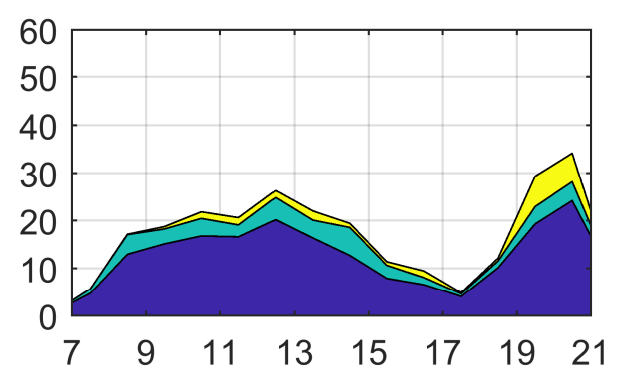

f)

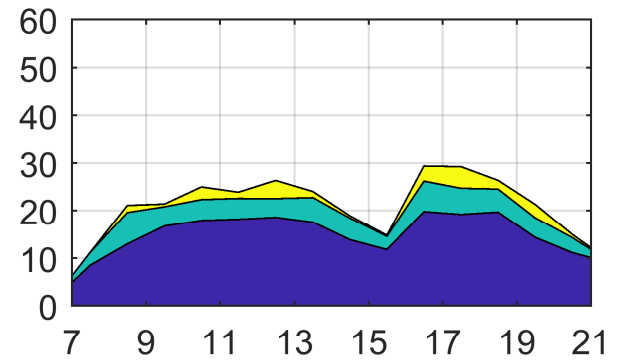

h)

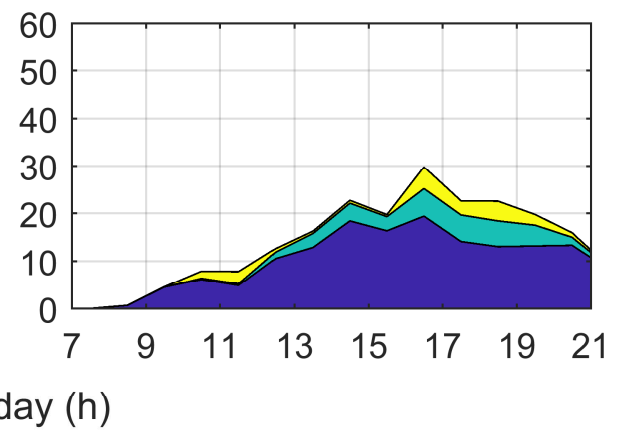

Figure 1. 


\begin{tabular}{|c|c|c|c|c|c|c|}
\hline & & & LPA & MPA & VPA & MVPA \\
\hline \multirow[t]{7}{*}{ 1st half } & Training day & Mean & 94 & 26 & 12 & 38 \\
\hline & & SD & 35 & 17 & 10 & 23 \\
\hline & Non-training day & Mean & 104 & 26 & 9 & 36 \\
\hline & & SD & 34 & 15 & 6 & 17 \\
\hline & Difference & Mean & -10 & -0 & 2 & 2 \\
\hline & & $\pm 95 \% \mathrm{CI}$ & 12 & 7 & 4 & 8 \\
\hline & & $\mathrm{p}$ value & 0.112 & 0.888 & 0.145 & 0.627 \\
\hline \multirow[t]{7}{*}{ 2nd half } & Training day & Mean & 76 & 53 & 53 & 105 \\
\hline & & SD & 24 & 17 & 17 & 24 \\
\hline & Non-training day & Mean & 94 & 25 & 15 & 40 \\
\hline & & SD & 40 & 18 & 18 & 25 \\
\hline & Difference & Mean & -18 & 27 & 37 & 65 \\
\hline & & $\pm 95 \% \mathrm{CI}$ & 12 & 7 & 7 & 10 \\
\hline & & $\mathrm{p}$ value & 0.004 & 0.000 & 0.000 & 0.000 \\
\hline \multirow[t]{7}{*}{ Whole day } & Training day & Mean & 170 & 78 & 65 & 143 \\
\hline & & SD & 51 & 27 & 19 & 32 \\
\hline & Non-training day & Mean & 198 & 51 & 25 & 76 \\
\hline & & SD & 60 & 26 & 19 & 38 \\
\hline & Difference & Mean & -28 & 27 & 40 & 67 \\
\hline & & $\pm 95 \% \mathrm{CI}$ & 18 & 10 & 7 & 14 \\
\hline & & p value & 0.005 & 0.000 & 0.000 & 0.000 \\
\hline
\end{tabular}




\begin{tabular}{|l|l|l|c|c|c|c|}
\hline & & & \multicolumn{1}{|l|}{ LPA } & \multicolumn{1}{l|}{ MPA } & VPA & MVPA \\
\hline Whole day & Training day & Mean & 126 & 54 & 64 & 118 \\
\hline & & S.D. & 45 & 21 & 28 & 36 \\
\hline & Non-training day & Mean & 148 & 34 & 21 & 56 \\
\hline & & S.D. & 58 & 26 & 34 & 49 \\
\hline & Difference & Mean & -22 & 20 & 43 & 63 \\
\hline & & $\pm 95 \%$ CI & 30 & 19 & 26 & 36 \\
\hline & & p-value & 0.128 & 0.042 & 0.003 & 0.003 \\
\hline
\end{tabular}

\begin{tabular}{|llll|}
\hline & LPA before & MPA before & VPA before \\
\hline LPA practice & $\mathrm{r}=0.415^{* * *},[0.13,0.66] \mathrm{r}=0.338^{* *},[0.04,0.58]$ & $\mathrm{r}=0.369^{* *},[0.08,0.60]$ \\
MPA practice & $\mathrm{r}=0.745^{* * *},[0.57,0.85] \mathrm{r}=0.509^{* * *},[0.27,0.70]$ & $\mathrm{r}=0.426^{* * *},[0.14,0.64]$ \\
VPA practice & $\mathrm{r}=0.666^{* * *},[0.46,0.81] \mathrm{r}=0.633^{* * *},[0.41,0.78]$ & $\mathrm{r}=0.476^{* * *},[0.20,0.68]$ \\
\hline & & \\
& \\
\end{tabular}

\begin{tabular}{|llll|}
\hline & LPA before & MPA before & VPA before \\
\hline LPA practice & $r=0.482^{*},[-0.07,0.81]$ & $r=0.413,[-0.15,0.77]$ & $r=0.557^{* *},[0.04,0.84]$ \\
MPA practice & $r=0.837^{* * *},[0.55,0.95]$ & $r=0.542^{*},[0.02,0.83]$ & $r=0.781^{*},[0.43,0.93]$ \\
VPA practice & $r=0.668^{* * *},[0.21,0.88]$ & $r=0.539^{*},[0.01,0.83]$ & $r=0.470^{*},[-0.08,0.80]$ \\
\hline & & \\
\end{tabular}

